\title{
Analysis and Comparison of ARCH Effects \\ for Shanghai Composite Index and NYSE Composite Index
}

\author{
Xinghao Liao, Guangdong Qi \\ School of Finance, Shanghai University of Finance and Economics \\ Shanghai 200439, China \\ E-mail: liaoxh2006@163.com
}

\begin{abstract}
In this article, we used ARCH class model to analyze and compare the income fluctuations of Shanghai Stock Market and NYSE, and obtained the characters of fluctuation for two markets and the models which fitted to describe them. At the same time, we also analyzed the fluctuation overflow effect of two markets, validated the character that Chinese stock market was not relatively mature and possessed low international market integration degree, and put forward relative policy advances.
\end{abstract}

Keywords: Shanghai composite index, NYSE composite index, ARCH class model, Fluctuation overflow

\section{Introduction}

In the domain of finance, the data of time sequence always put up characters such as convergence and lever effect, but traditional method researching financial data could not consider these characters, which induce the predictive ability of model is bad. Since Engle put forward ARCH model in 1982 (Engle, 1982, pp. 987-1008), the domain of conditional heteroscedasticity developed very quickly, and many variations of ARCH model occurred, and the most representative model is the generalized ARCH model which was put forward by Bollerslev in 1986 (Bollerslev, 1986, pp. 309-327), i.e. GARCH model, and it is extensively applied in the financial research and actual operation.

Since 2005, as the emerging market, Chinese stock market has been more and more concerned by the world, and in less than two years, the stock market values of Shanghai and Shenzhen was doubling more. The increase speed makes most markets in the world amazing. However, there are many gaps between Chinese markets with international financial market, which is undeniable, for example, the financial products in Chinese capital market is relatively few, and the structure is too single, and the fluctuation is large, and the behaviors of market control still exist. Comparing with that, in those mature international capital markets such as the capital markets in UN, US and many developed capitalism countries, the fluctuation is small, and the financial products is very abundant, the market order is good and the financial supervision system is mature. In this article, we will use the NYSE as the representation of international mature financial market and take Shanghai Stock Exchange as the representation of Chinese markets, analyze and compare the fluctuation of daily yield for two markets composite index to try to find out the deficiencies and reasons existing in Chinese stock market relative to the mature market.

\section{Introduction of ARCH class of model}

One obvious character of the financial time sequence is the conditional heteroscedasticity, and the ARCH class of model has been extensively applied in the domain of financial measurement. GARCH model is most extensively adopted in the research of fluctuation, which is defined by the average value equation and the conditional variance.

\subsection{Model of ARCH}

$\mathrm{ARCH}$ model is the simplest ARCH class of model, and it supposes the conditional variance of $\mathrm{t}$ term is the function of residual error from t-1 term to t-q term, i.e. the fluctuation is self-correlative, and the form of ARCH (q) is

$\sigma_{t}^{2}=\alpha_{0}+\sum_{i=1}^{q} \alpha_{i} \varepsilon_{t-i}^{2}$

and the non-negative condition is $\alpha_{0}>0, \alpha_{i} \geq 0(i=1,2, \cdots, q)$.

\subsection{Model of GARCH}

The GARCH model is generalized ARCH model, and it supposes the conditional variance is former conditional 
variance based on the simple ARCH model, i.e. the conditional variance is self-correlative, and the form of GARCH (p, q) is

$$
\sigma_{t}^{2}=\alpha_{0}+\sum_{i=1}^{q} \alpha_{i} \varepsilon_{t-i}^{2}+\sum_{j=1}^{p} \beta_{j} \sigma_{t-j}^{2}
$$

Where, $p \geq 0, q>0$, the non-negative condition is $\alpha_{0}>0, \alpha_{i} \geq 0(i=1,2, \cdots, q), \beta_{j} \geq 0(j=1,2, \cdots, p)$, and to make the conditional variance stable, it must fulfill the condition (Bollerslev, 1986, P.309-327),

$\sum_{i=1}^{q} \alpha_{j}+\sum_{j=1}^{p} \beta_{i}<1$

and its value reflects the durative of the fluctuation.

\subsection{Model of GJR-GARCH}

GJR-GARCH model (Glosten, 1993, P. 1779-1801) overcomes the limitation that GARCH model cannot describe the positive and negative reaction of collision, and its form is

$\sigma_{t}^{2}=\alpha_{0}+\sum_{i=1}^{q} \alpha_{i} \varepsilon_{t-i}^{2}+\sum_{j=1}^{p} \beta_{j} \sigma_{t-j}^{2}+\gamma \varepsilon_{t-1}^{2} I_{t-1}$

where, $I_{t-1}=\left\{\begin{array}{l}1\left(\varepsilon_{t-1}<0\right) \\ 0\left(\varepsilon_{t-1} \geq 0\right)\end{array}\right.$, and under the same limitation condition with GARCH model, the non-negative condition also requires $\alpha_{1}+\gamma \geq 0$.

\subsection{Model of EGARCH}

Like GJR-GARCH model, the EGARCH model also could be used to deal with the time sequence obtained by the lever effect. It was put forward by Nelson in 1991 (Nelson, 1991, P. 347-370), and its form is

$\log \left(\sigma_{t}^{2}\right)=\alpha_{0}+\sum_{i=1}^{p} \beta_{i} \log \left(\sigma_{t-i}^{2}\right)+\sum_{j=1}^{q} \alpha_{j}\left(\left|\frac{\varepsilon_{t-j}}{\sqrt{h_{t-j}}}-\sqrt{\frac{2}{\pi}}\right|+\gamma_{j} \frac{\varepsilon_{t-j}}{\sqrt{h_{t-j}}}\right)$

In a comparison among ARCH, GARCH, EGARCH and other more complex half-parameter and zero-parameter models, Pagan and Schwert (Pagan, 1990, P. 267-290) proved that simple EGARCH $(1,1)$ could implement modeling to the financial time sequence.

\section{Data selection and basic analysis}

In this article, we select the data of Shanghai Stock Exchange from Jan 10, 2003 to Dec 28, 2007, and to make the analysis possess comparison, the data of NYSE are also in the same period. The backgrounds include that Chinese economy had been developed very quickly since China joined in WTO, and US basically cast off the shadow of 9.11 in 2003 and its economy begun to recover to stable status. The computation formula of yield used in the article is $r_{t}=100 \times \ln \left(p_{t} / p_{t-1}\right)$, where, $r_{t}$ is the daily logarithm yield (\%), $p_{t}$ and $p_{t-1}$ are respectively the index values in $\mathrm{t}$ term and $\mathrm{t}-1$ term.

The descriptive stat. results of two markets are seen in Table 1.

The daily yield average of Shanghai composite index is $0.11 \%$, and the daily yield average of NYSE composite index is $0.05 \%$, and the former is twice than the latter, which indicates as the emerging market, the yield of China stock market is higher, and it also explains why international idle money continually has flowed into China in recent years. In addition, we can obviously see that large fluctuation existed in both markets, and the result showed negative deflection, and the kurtosis exceeded 3, which indicated the yield distributions of two markets were non-normal, and comparing with NYSE composite index, Shanghai composite index had more obvious and larger deflection and higher kurtosis, so the possibility that the yield of Shanghai Stock Exchange kept away from the average was higher, and higher risk existed in Shanghai stock market, and the market was not stable and large deflection would always occur in the party with negative yield. From the descriptive statistic, as the emerging market, the yield of Shanghai Stock Exchange was obviously higher than such mature market as NYSE, and the market of Shanghai was not mature like NYSE, and large fluctuation existed in it.

Implement ADF test to the daily yield sequence of samples. The test result showed the ADF test $t$ stat. of Shanghai composite index daily yield sequence is -14.3858 under the significance level of $1 \%$, and it was obviously lower than 
the critical of -3.4386 under the significance level of $1 \%$. The ADF test $t$ stat. of NYSE composite index daily yield sequence is -15.6572 under the significance level of $1 \%$, and it was obviously lower than the critical of -3.4386 under the significance level of $1 \%$. So we reject the original hypothesis, i.e. two groups of daily yield sequence have no unit root, and they are stable sequence.

We implement self-correlative and deviated self-correlative coefficient analysis with 25-order to Shanghai composite index and NYSE composite index, and the results showed that in all time lags, the self-correlative function value and the deviated self-correlative function value of yield were small, and they were smaller than 0.07 , so the daily yield sequences of Shanghai composite index and NYSE composite index were not self-correlative.

Implement self-regression with 10-order lag items to the yields of two markets, and test ARCH effect to the residual error. For the Shanghai composite index, the F statistic of ARCH effect test is 9.965872, and the LM statistic is 48.04335. For the NYSE composite index, the F statistic of ARCH effect test is 18.95560 , and the LM statistic is 88.36852. The statistics of two markets are very significant, which indicate the ARCH effect exists in the daily yield data of Shanghai composite index and NYSE composite index.

\section{Fluctuation analysis of ARCH model}

Implement modeling to the yield sequence, and respectively use ARCH (1), GARCH $(1,1)$, GJR-GARCH $(1,1)$ and EGARCH $(1,1)$ models to describe the daily yield heteroscedasticity of Shanghai composite index and NYSE composite index, and the estimation results are seen in Table 2 (values in the bracket are $t$ statistics).

From Table 2, the $\beta_{1}$ coefficients of GARCH model cluster are big and pass the significant test, which indicates the stock price fluctuation possesses "long-term memory", i.e. the fluctuation of past price is correlative with the fluctuation of infinite term price. In the equation of conditional variance, the coefficients $\alpha_{1}$ and $\beta_{1}$ are significantly positive, which indicates past fluctuation has positive and released influence to the future fluctuation of the market, so the clustered phenomena occurs in the stock market fluctuation. $\alpha_{1}+\beta_{1}$ is closed to 1 , which indicates the reaction function of stock fluctuation to the exterior concussion is degressive by a relatively slow speed, and the big fluctuation in the stock market cannot be eliminated in the short term. In addition, because $\alpha_{1}+\beta_{1}$ in ARCH $(1)$, GARCH $(1,1)$ and GJR-GARCH $(1,1)$ are smaller than 1 , so the conditional variance sequence of yield is stable, and the model can be predicted.

Estimate two markets by the GJR-GARCH $(1,1)$ model, we find the yield of Shanghai composite index has no obvious lever effect, but the negative concussion brings smaller fluctuation than the positive concussion. It also indicates under immature market, investors are not rational enough, and higher average yield and big fluctuation will induce more short-term investors, and when the market possesses big positive yield, investors will close out and induce big fluctuation. Comparing with Shanghai composite index, NYSE composite index has obvious lever effect, and its behavior is consistent with our anticipation to the mature market.

\section{Explanation degree comparison of various models}

For the regression result, every model has certain limitation, but we can try to find out the best models for two markets. The proofs to judge the model include information principle, F statistic and logarithm maximum likelihood method. But for the nonlinear model, the maximum likelihood method is the best choice, so we adopt the logarithm maximum likelihood method to rank the model, and the results are seen in Table 3.

From the information principle method and the maximum likelihood method, for the Shanghai stock market, the best fluctuation descriptive model is EGARCH model, which is consistent with Liqing and Zhao, Lijia's conclusions ( $\mathrm{Li}$, 2004, pp. 31-32), and though the symbols of the asymmetric item don't accord with the anticipation, but maybe the unconformity just indicates the characters of Chinese stock market, i.e. bigger positive yield will bring bigger fluctuation. For the NYSE stock market, the best model is GJR-GARCH, which indicates NY stock market has strong lever effect which doesn't exist in Shanghai stock market.

\section{Overflow effect of Shanghai and Shenzhen Stock Markets fluctuation}

Estimate Shanghai composite index by EGARCH model and estimate NYSE composite index by GJR-GARCH model, and respectively obtain the conditional variance data sequence GARCH01 and GARCH02 of the residual item, and implement Granger causality test to both sequences and the results are seen in Table 4.

From the test results, we can see that the influence of Shanghai stock market on New York stock market is not notable, and the influence of New York stock market on Shanghai stock market is small, which indicates though the market value of Chinese stock market, but after all the capital market of China is not opening, so the influences whether from interior to exterior or from exterior to interior is very limited, and the investment of QDII would obviously reduce the investment risk of domestic investors. 


\section{Conclusion}

To compare the daily yields of two markets by the modeling of ARCH class model, we can see that the lever effect almost doesn't exist in Shanghai stock market, but the lever effect of NYSE stock market is more obvious, which reasons are mainly that Chinese government often intervenes the stock market and stabilize the price, and it also indicates participators' gambling attitudes are strong. At the same time, from the analysis of ARCH-M class model, we can see that Chinese investors could obviously avoid the risk and require higher return for the investment with high risk, but US investors have not same strong avoidance degree to the risk comparatively.

The test result of fluctuation overflow effect is that the fluctuation overflow effect of two markets is not obvious, and the influence of Chinese stock market on NYSE stock market is stronger. Of course, the Granger causality test only checks up the statistical causality relationship and it doesn't indicate the causality relationship with actual meanings certainly exist or doesn't exist in both markets. And the conclusion only could be referred.

As the emerging market, the average income of Shanghai stock market is higher than NYSE stock market, but the stronger fluctuation also indicates Chinese investors' investment concept is not strong, their investment behaviors are easily suffered by all kinds of information, and the Shanghai stock market is not mature. These fluctuation characters of Shanghai stock market could provide decision-making references to avoid risks for investors and implement market supervision for the management department. In addition, the big fluctuations of Chinese stock market are mainly induced by the policy interferences of management department, and most so-called concussions belong to policy concussion. Therefore, the management department should be more careful when coming on policies, really realize "information symmetry", hold well the adjustment degree of policy, and consider the market control from long-term view to make the policy more reasonable and consecutive.

\section{References}

Bollerslev T. (1986). Generalized Autoregressive Conditional Heteroskedasticity. Journal of Econometrics, No. 31, pp. 309-327.

Engle R F. (1982). Autoregressive Conditional Heteroskedasticity with Estimates of the Variance of UK inflation. Econometrica, No. 50, pp. 987-1008.

Glosten L R, Jagannathan R. and Runkle D E. (1993). On the Relation between the Expected Value and Volatility of the Nominal Excess Return on Stocks. The Journal of Finance, No. 48(5), pp. 1779-1801.

Li Q and Zhao L J. (2004). The Applied Research of the ARCH Model in the Stock Market in China. Xi'an Finance, No. 11, pp. 31-32.

Nelson D B. (1991). Conditional Heteroskedasticity in Asset Returns: A New Approach. Econometrica, No. 59(2), pp. 347-370.

Pagan A R and Schwert G W. (1990). Alternative Models for Conditional Stock Volatility. Journal of Econometrics, No. 45(12), pp. 267-290.

Table 1. Descriptive Stat. of two markets income

\begin{tabular}{|c|c|c|c|c|c|c|c|}
\hline \multicolumn{7}{|c|}{ Descriptive Stat. of Shanghai composite index income } \\
\hline Average & Median & Max. & Min. & $\begin{array}{c}\text { Standard } \\
\text { deviation }\end{array}$ & Deflection & Kurtosis & Jarque-Bera \\
\hline 0.114430 & 0.094522 & 7.890344 & -9.256154 & 1.534343 & -0.324148 & 6.400324 & 603.1188 \\
\hline \multicolumn{7}{|c|}{ Descriptive Stat. of NYSE composite index income } \\
\hline Average & Median & Max. & Min. & $\begin{array}{c}\text { Standard } \\
\text { deviation }\end{array}$ & Deflection & Kurtosis & Jarque-Bera \\
\hline 0.050530 & 0.088661 & 3.087640 & -3.596156 & 0.818359 & -0.276442 & 4.711740 & 159.0871 \\
\hline
\end{tabular}


Table 2. Estimation results of ARCH class model

\begin{tabular}{|c|c|c|c|c|c|}
\hline \multicolumn{6}{|c|}{ Daily yield of Shanghai composite index } \\
\hline & $\alpha_{0}$ & $\alpha_{1}$ & $\beta_{1}$ & $\gamma$ & $\alpha_{1}+\beta_{1}$ \\
\hline \multirow[t]{2}{*}{$\mathrm{ARCH}(1)$} & 1.972974 & 0.135552 & & & \\
\hline & $(26.671)$ & $(4.527)$ & & & \\
\hline \multirow[t]{2}{*}{ GARCH $(1,1)$} & 0.032168 & 0.066397 & 0.921614 & & 0.988011 \\
\hline & $(2.651)$ & $(5.866)$ & $(69.822)$ & & \\
\hline \multirow[t]{2}{*}{ GJR-GARCH $(1,1)$} & 0.031706 & 0.068466 & 0.922838 & 0.006445 & 0.991304 \\
\hline & $(2.623)$ & $(4.586)$ & $(69.151)$ & $(-0.402)$ & \\
\hline \multirow[t]{2}{*}{ EGARCH $(1,1)$} & -0.094005 & 0.140626 & 0.983796 & 0.01092 & 1.124422 \\
\hline & $(-5.794)$ & $(6.029)$ & $(160.997)$ & $(1.053)$ & \\
\hline \multicolumn{6}{|c|}{ Daily yield of NYSE composite index } \\
\hline & $\alpha_{0}$ & $\alpha_{1}$ & $\beta_{1}$ & $\gamma$ & $\alpha_{1}+\beta_{1}$ \\
\hline \multirow[t]{2}{*}{$\mathrm{ARCH}(1)$} & 0.595352 & 0.075448 & & & \\
\hline & $(27.339)$ & $(3.111)$ & & & \\
\hline \multirow[t]{2}{*}{ GARCH $(1,1)$} & 0.012961 & 0.050941 & 0.927192 & & 0.978133 \\
\hline & $(3.287)$ & $(5.079)$ & $(62.217)$ & & \\
\hline \multirow[t]{2}{*}{ GJR-GARCH $(1,1)$} & 0.013376 & -0.005523 & 0.937436 & 0.087154 & 0.931913 \\
\hline & $(3.898)$ & $(-0.536)$ & $(65.065)$ & $(5.329)$ & \\
\hline \multirow[t]{2}{*}{ EGARCH $(1,1)$} & -0.078879 & 0.086468 & 0.977993 & -0.078487 & 1.064461 \\
\hline & $(-4.176)$ & $(5.834)$ & $(158.229)$ & $(-5.457)$ & \\
\hline
\end{tabular}

Table 3. Ranking models by logarithm maximum likelihood method

\begin{tabular}{|c|c|c|c|c|c|}
\hline \multicolumn{7}{|c|}{ Shanghai composite index } \\
\hline & Model & Log likelihood & AIC & F-statistic & Prob(F-statistic) \\
\hline 1 & EGARCH & -2082.445 & 3.534536 & 4.105031 & 0.000033 \\
\hline 2 & GJR-GARCH & -2084.747 & 3.538425 & 4.229756 & 0.000021 \\
\hline 3 & GARCH & -2084.807 & 3.536836 & 4.751788 & 0.000009 \\
\hline 4 & ARCH & -2154.182 & 3.652334 & 5.504430 & 0.000003 \\
\hline \multicolumn{7}{|c|}{ NYSE composite index } & & F-statistic & Prob(F-statistic) \\
\hline & Model & Log likelihood & AIC & 2.599255 & 0.008079 \\
\hline 2 & GJR-GARCH & -1382.519 & 2.264473 & 2.668622 & 0.006595 \\
\hline 3 & EGARCH & -1387.817 & 2.273095 & 2.978264 & 0.004217 \\
\hline 4 & GARCH & -1394.579 & 2.282472 & 4.114241 & 0.000426 \\
\hline
\end{tabular}

Table 4. Fluctuation overflow effect test of two markets (Granger causality test)

\begin{tabular}{|c|c|c|}
\hline Zero-hypothesis & F-Statistic & Probability \\
\hline GARCH02 is not the Granger01 reason of GARCH01 & 1.38297 & 0.12075 \\
\hline GARCH01 is not the Granger02 reason of GARCH01 & 1.46983 & 0.08301 \\
\hline
\end{tabular}

